www.jmscr.igmpublication.org

Impact Factor (SJIF): 6.379

Index Copernicus Value: 79.54

ISSN (e)-2347-176x ISSN (p) 2455-0450

crossrefDOI: https://dx.doi.org/10.18535/jmscr/v6i10.185

Journal Of Medical Science And Clinical Research

\title{
Clinical manifestations, comorbidities and causative organisms of infections in children aged 6 months to 59 months with Severe Acute Malnutrition
}

\section{Authors \\ Dr Pravati Jena, Dr Soumini Rath, Dr Manas Kumar Nayak, Dr Diptirekha Satapathy, Dr Palash Das}

Department of Pediatrics, Kalinga Institute of Medical Sciences, KIIT Deemed to be University, Bhubaneswar, 751024, Odisha

Corresponding Author

Dr Soumini Rath

Department of Pediatrics, Kalinga Institute of Medical Sciences, KIIT Deemed to be University,

Bhubaneswar, 751024 Odisha, India

Mobile no 9337799393, Email: soumini19@gmail.com

\begin{abstract}
Objective: To study the various clinical manifestations, co-morbidities and causative organisms of infections in SAM (severe acute malnutrition) in children aged 6 months to 59 months in a tertiary care hospital of odisha.
\end{abstract}

Methods: It is a hospital based prospective observational study done in a tertiary care hospital, Cuttack, Odisha during the period of November 2015 to October 2017.All children from 6 months to 59 months of age diagnosed with SAM were included and the clinical manifestations and spectrum of infections in them were evaluated.

Results: In our study population the prevalence of SAM was found to be $2.8 \%$.Fever was the most common symptom (71\%), next was vomiting (51\%), loose stools (46.8\%), cough $46.3 \%$ and poor appetite and/ or weight loss (31\%).Most common comorbidities found were diarrhea(46.8\%), acute respiratory tract infection (46.3\%), septicemia(27.4\%) and urinary tract infection(26.3\%). The commonest organisms encountered in blood culture in the study were Staphylococcus aureus (48.1\%) followed by CONS (28.9\%), Group D Streptococcus (11.5\%), E.Coli (7.7\%), and Klebsiella (3.8\%).

Conclusion: Severe acute malnutrition is prevalent in odisha (2.8\%) and leads to increased Mortality (6.8\%) in children between 6 months to 59 months of age. The commonest presentation to hospital is fever(71\%) with comorbid condition acute gastroenteritis(46.8\%) followed by respiratory tract infections and septicemia. The most common infective organism affecting children with SAM is staphylococcus aureus(48.1\%) followed by CONS (28.9\%).

Keywords: SAM (severe acute malnutrition), infections, clinical manifestations, comorbidities, Odisha.

\section{Introduction}

Malnutrition is one of the most leading causes of morbidity and mortality in children all over the world. The most extreme form of undernutrition is
SAM (severe acute malnutrition). Severe acute malnutrition (SAM) is defined as weight for height/length less than $-3 \mathrm{Z}$ score of the WHO growth charts or MUAC $<115 \mathrm{~mm}$ or the presence 
of bilateral pitting edema. ${ }^{(1)}$ Some of the important contributory factors for SAM are lack of exclusive breastfeeding, delayed introduction of complementary feeds, feeding diluted feeds, repeated enteric and respiratory tract infections, illiteracy, and low socioeconomic status. ${ }^{(2)}$ As per National Family Health Survey (NFHS)-4 (201516) $35.7 \%$ children below five years are underweight, 38.4\% are stunted and $21 \%$ are wasted in India. Stunting and underweight prevalence has decreased since 2005-06, especially for stunting, which declined from 48 percent in 2005-06 to 38 percent in 2015-16 but the prevalence of wasting has remained about the same. $^{(3)}$ Children with malnutrition usually present with fever, loss of appetite or with comorbid conditions like gastroenteritis and respiratory tract infections followed by urinary tract infections and septicemia ${ }^{(4,5)}$ There is an increased severity of common infectious diseases in children with SAM, leading to increased mortality in them.

Management of infections differ in malnourished children as compared to healthy children. ${ }^{(6)}$ Organisms most commonly affecting malnourished children are Staphylococcus aureus, Escherichia coli , and Salmonella typhi. ${ }^{(7)}$ Thus malnutrition and infection can be mutually aggravating and produces more serious consequence for the patient. A good number of SAM cases are admitted to our hospital from all over Odisha with variety of infections like RTI, diarrhoea, measles, tuberculosis, UTI etc, who may or may not have signs \& symptoms of overt infection. However the clinical profile and incidence of bacteremia in malnourished children has not been adequately studied in recent past in Odisha, so this study was conducted to know the spectrum of infection and causative organisms among severe acute malnutrition cases admitted to this institution.

\section{Materials and Methods}

This study is a prospective observational study of patients admitted with severe acute malnutrition in the department of pediatrics in a tertiary care hospital of Odisha during the period of November 2015 to October 2017.

Inclusion Criteria: Children from 6 months to 59 months of age with

- Weight-for-height less than -3 SD (WHO/ NCHS median height) and/or

- Visible severe wasting and/or

- Mid arm circumference (MUAC) < 11.5 $\mathrm{cm}$ and/or

- Bilateral pedal edema

\section{Exclusion Criteria}

- Children < 6 month and >5 yrs

- Malnutrition due to chronic illness (secondary malnutrition) like cleft lip, cleft palate, GERD, pyloric stenosis and other surgical condition, chronic renal failure, congenital heart diseases, liver disorders, asthma, mental retardation, cerebral palsy, suspected case of inborn error of metabolism etc.

Children whose parents denied for consent were also excluded from the study.

The parents of the children who satisfied the inclusion criteria were informed and a written consent was taken from them. This study was approved by the Ethics and Research Committee of our hospital.

\section{Demographic Details and Clinical Information}

A detailed history of all cases with reference to nutritional history, SES, contact with TB, history of diarrhea and other infectious diseases. Birth history, family order, duration of breastfeeding, immunization, weaning and duration of illness were taken. A thorough examination was carried out along with different anthropometric measurements expressed in standard deviation from the median of the reference population standards (NCHS). Clinical evidence of associated infections were considered. Data regarding residential area, sex, age and date of birth, socioeconomic status, perinatal history, feeding practice was collected by questionnaire method. Proper history of all children suffering from 
various presentations were taken and questioned and data regarding frequency, nature and duration of diarrhea and vomiting and fever along with cough and other symptoms were recorded. Other associated infections like urinary tract infection, pneumonia, septicemia, TB, malaria and HIV were assessed.

History of chronic diseases such as sickle cell disease, HIV/AIDS and TB was also enquired. All the evidence and finding were recorded in predefined performa and data were analyzed statistically.

\section{General Examination}

A thorough clinical examination was done on all the enrolled patients and severe acute malnutrition was diagnosed as per WHO criteria. The measurements done were as follows:

\section{Weight}

Children $<2$ yrs of age were weighed using a 25 $\mathrm{kg}$ Salter hanging scale (CMS Weighing equipment, High Holborn, London United kingdom) and those $>2$ yrs of age were weighed while standing on the measuring board. Before each recording the scales were adjusted to zero. Weight was rounded off to nearest 100 grams and measured daily during morning. The reference which was applied was of WHO chart which compared weight/length or weight/height.

\section{Length / Height}

- For children<2 yrs length was measured using an infantometer in the recumbent position by two examiners. For $>2$ yrs children and able to walk, height was measured while standing using a stadiometer.

- Weight for height/length and Z score of less than -3SD were included in the study.

\section{Mid-Upper Arm Circumference (MUAC)}

For measurement of MUAC a midpoint between acromion process and olecranon process was chosen on the left arm. The reading was recorded to the nearest $0.1 \mathrm{~cm}$.MUAC $<11.5 \mathrm{~cm}$ were considered as severe acute malnutrition. MUAC can be measured above 6 months of age.

\section{Specimen Collection and Laboratory Procedures}

Investigations which were done at SCB Medical College and SVPPGIP main laboratory were CBC, CPS, ESR, Blood slide for malaria parasite, serum total protein and albumin, serum electrolytes, x-ray chest, urine and stool examination, test for HIV. Culture and sensitivity testing of blood, urine, stool and pus were done. Random blood glucose estimation was done in the ward.

\section{Data Analysis}

Data were entered into a computer excel 2007 using IBM SPSS 21 and Microsoft. Data was analyzed using SPSS software. The 95\% confidence interval was determined. Factors/ predictors with $\mathrm{p}$-value of less than 0.05 were considered significant. Data were double entered into an Access database and checked for errors

\section{Results}

A total of 311 patients enrolled in the study between 6 month to 59 month of age during this period of 2 years (November 2015 to October 2017). Out of these 102 patients were excluded as per exclusion criteria and 19 patients left against medical advice during study period. So the remaining 190 patients constituted the study population.

Out of total 10878 patients admitted to our hospital during the 2 years of study period, $311 / 10878(2.8 \%)$ patients were having features of SAM. (Table No-1)

The commonest clinical symptom in the present study was fever $71 \%$, then vomiting $51 \%$, loose stools $46.8 \%$,cough $46.3 \%$ and poor appetite/or weight loss in $31 \%$ of cases. Other presenting complaints were oedema $14.7 \%$, distension of abdomen in $13.1 \%$, rash $4.2 \%$, convulsion $3.1 \%$, ear discharge $2.1 \%$ and bleeding 1.6\%.(TABLE NO-2) 52 of the blood cultures collected grew bacteria out of which 25(48.1) grew Staphylococcus aureus, 15(28.9\%) grew CONS, all being Staphylococcus epidermidis, 6(11.5\%) grew Group D streptococcus, 4(7.7\%) E.Coli and 
Klebsiella species in 2(3.8\%). Gram positive organisms were more common in the above findings (Table No -3) The most common comorbidities associated with severe acute malnutrition were acute gastroenteritis in $89 / 190$ (46.8\%), followed by acute respiratory tract infection in $88 / 190(46.3 \%)$, septicaemia in $52 / 190(27.4 \%)$ urinary tract infection in 50/190 (26.3\%), malaria in $8 / 190(4.2 \%)$ patients. Other associated infections were tuberculosis in 5/ 190 (2.6\%), measles in $5 / 190$ (2.6\%), meningitis 4/190 (2.1) otitis media in 4/190 (2.1\%) and HIV in $1 / 190(0.5 \%)$. Other infection skin infection like pyoderma, scabies, candidiasis are found in variable number of patient were 21/190 (11\%), $6 / 190(3.1 \%)$ and $4 / 190(2.1 \%)$ respectively. (Table No-4)

Table-1: Prevalence of SAM in the Study Population

\begin{tabular}{|l|c|c|}
\hline $\begin{array}{l}\text { Total No } \\
\text { of Patient }\end{array}$ & $\begin{array}{c}\text { No. of Sam Patients } \\
(\boldsymbol{\%})\end{array}$ & $\begin{array}{c}\text { Others } \\
(\boldsymbol{\%})\end{array}$ \\
\hline 10878 & $311(2.8)$ & $10,567(97.2)$ \\
\hline
\end{tabular}

Table No 2: Distribution of the Study Population as Per Major Present Complaint $(n=190)$

\begin{tabular}{|c|c|c|c|}
\hline S. NO. & Complaints & Frequency & $\begin{array}{c}\text { Percentage } \\
(\boldsymbol{\%})\end{array}$ \\
\hline 1. & Fever & 135 & 71 \\
\hline 2. & Vomiting & 97 & 51 \\
\hline 3. & Loose Motion & 89 & 46.8 \\
\hline 4. & Cough & 88 & 46.3 \\
\hline 5. & $\begin{array}{c}\text { Loss of } \\
\text { Appetite/ } \\
\text { (Weight Loss) }\end{array}$ & 59 & 31 \\
\hline 6. & Edema & 28 & 14.7 \\
\hline 7. & $\begin{array}{c}\text { Abdominal } \\
\text { Distension }\end{array}$ & 25 & 13.1 \\
\hline 8. & Rash & 8 & 4.2 \\
\hline 9. & Convulsion & 6 & 3.1 \\
\hline 10. & Ear Discharge & 4 & 2.1 \\
\hline 11. & Bleeding & 3 & 1.6 \\
\hline
\end{tabular}

Table-3: Type and Proportion of Bacteria Isolated From Blood Culture $(n=52)$

\begin{tabular}{|l|c|c|}
\hline Organism & Frequency & $\begin{array}{c}\text { Percentage } \\
(\boldsymbol{\%})\end{array}$ \\
\hline Staph.Aureus & 25 & 48.1 \\
\hline CONS & 15 & 28.9 \\
\hline GDS & 6 & 11.5 \\
\hline E.Coli & 4 & 7.7 \\
\hline Klebsiella Species & 2 & 3.8 \\
\hline Total & 52 & \\
\hline
\end{tabular}

Table No 4: Associated Co-Morbidities in the Study Population (n-190)

\begin{tabular}{|l|c|c|}
\hline Type of Diseases & Frequency & $\begin{array}{c}\text { Percentage } \\
\text { \% }\end{array}$ \\
\hline Acute Gastroenteritis & 89 & 46.8 \\
\hline ARI & 88 & 46.3 \\
\hline Sepsis & 52 & 27.4 \\
\hline UTI & 50 & 26.3 \\
\hline Malaria & 8 & 4.2 \\
\hline Measles & 5 & 2.6 \\
\hline TB & 5 & 2.6 \\
\hline Meningitis & 4 & 2.1 \\
\hline Otitis Media & 4 & 2.1 \\
\hline HIV & 1 & 0.5 \\
\hline Skin Infections & 21 & 11 \\
\hline Pyoderma & 6 & 3.1 \\
\hline Candidiasis & 4 & 2.1 \\
\hline Scabies
\end{tabular}

\section{Discussion}

The prevalence of severe acute malnutrition in our study population was $2.8 \%(311 / 10878)$.According to NFHS-4 (2015-2016) data the overall prevalence of severe acute malnutrition in odisha is around 6.4\% which is much more than our results which may be due to poor referral, ignorance and lack of access to health services in some parts of the state. Mahgoub et al found prevalence of SAM to be $27.3 \%$ in a study done in Sudan which is significantly high ${ }^{(8)}$ which may be due to geographical and racial variation among study population.(Table No 1)

In our study, the most common clinical symptom was fever $(71 \%)$ followed by vomiting $(51 \%)$, loose stools (46.8\%), cough(46.3\%) and poor appetite and /or weight $\operatorname{loss}(31 \%)$.Other features were edema(14.7\%), abdominal distension (13.1\%), rash (4.2\%), convulsion(3.1\%),ear discharge $(2.1 \%)$, and bleeding(1.6\%).

In 2008 Bernal et al conducted a study on treatment of SAM in children by implementing the WHO Guidelines in which fever $(26.3 \%)$ was the most common symptom in $26.3 \%$ cases $^{(5)}$ and most common comorbid condition was diarrhea $(68.4 \%)$ and it was significantly higher in children with severe acute malnutrition $(\mathrm{P}=0.003)$.

Another study done by Bagga et al in 2003 also found fever and diarrhea as the most common presentations. $^{(4)}$ Other studies which showed 
similar results were by Ashraf et al in 2001 which reported diarrhea (25.8\%) and fever/vomiting $(30.9 \%)$ as the common presenting symptoms in malnourished children ${ }^{(9)}$.(Table No-2)

Out of 52 blood culture samples which grew bacteria 25(48.1\%) were Staphylococcus aureus, 15(28.9\%) grew CONS, all being Staphylococcus epidermidis, $6(11.5 \%)$ grew group D streptococcus, 4 (7.7\%) were E.Coli and 2(3.8\%) were Klebsiella species. Gram+ve aerobes were the most common isolates accounting for $88.5 \%$ (46/52) followed by the Gram - ve aerobes $11.5 \%$ (6/52). Umma Abdullahi Idris et al (2018) in his study found that Staphylococcus aureus (41.5\%), Escherichia coli $(17.1 \%)$, and Salmonella typhi (12.2\%) were the most frequent isolates in the individuals with severe malnutrition ${ }^{(7)}$. S.aureus predominance in this study may be due to the fact that it reflected the community predominant organism as most children were from community. There has been variable associations between malnutrition and risk of bacteremia. Previous studies from kenya have suggested an increased likelihood of Gram negative bacteraemia in children with malnutrition ${ }^{(10)}$. Vitamin A deficiency may be a predisposing factor to Staphylococcus aureus infections through phagocyte dysfunction and decreased complement activity $^{(11)}$. Other risk factor that could have contributed to the high prevalence of Staphylococcus aureus includes prior hospitalization as majority of the study participants were referred from peripheral hospital. Coagulase Negative Staphylococcus (CONS) was the second leading cause of bacteremia in this study. Studies from Africa and Jamaica have found CONS as the predominant organism ranging between 26.7 to $40 \%^{(12,13)}$. In our study the proportion of CONS isolates was $32.5 \%$. which may have been due to the use of $70 \%$ alcohol alone to clean the skin prior to venepuncture, rather than using it in combination with $10 \%$ povidone iodine as in other studies $(4,13,14)$. CONS are common commensals of skin and can enter the bloodstream if there is breech in barrier as in malnutrition ${ }^{(12)}$. CONS are recognized as important cause of sepsis among critically ill and immunocompromised children. ${ }^{(12,13)}$. This makes judging the clinical significance of CONS very important before considering them as contaminants. Group D Streptococcus bacteria were another Gram - positive blood bacterial isolates in this study. It accounted for $11.5 \%$ of all isolates. Group D Streptococcus are normal commensals of the gastrointestinal tract of humans. In malnourished children there occurs damage to the gastrointestinal mucosa and impaired immune response which leads to infection with these bacteria. E.coli and klebsiella species were the Gram - negative isolates that were demonstrated by this study. They accounted for $7.7 \%$ and $3.8 \%$ of all isolates respectively. Alem et al (2011) in Ethiopia ${ }^{(15)}$ and Christie et al (1992) (12) in Jamaica also reported similar results.(Table No-3)

The most common comorbidities associated with SAM were AGE (46.8\%), followed ARI (46.3\%), septicemia (27.4\%), UTI (26.3\%), malaria (4.2\%) and other associated co-infection were tuberculosis $(2.6 \%)$ measles $(2.6 \%)$, otitis media (2.1\%), HIV $(0.5 \%)$ and skin infection like pyoderma, candidiasis and scabies. Prasanth et al (2018) reported pneumonia as the major co morbid condition in $43 \%$ of cases. Their study reported diarrhea in $21 \%$ of cases ${ }^{(16)}$. Our study had similar as done by Sharma et $\mathrm{al}^{(17)}$ and Bernal et $\mathrm{al}^{(5)}$ who reported diarrheas as the major comorbid condition in malnourished children. (Table No-4)

\section{Summary}

- The overall prevalence of SAM in our study population was $2.8 \%$.

- Fever was the most common clinical feature associated with SAM.

- Commonest comorbidities associated with SAM are diarrhoea followed by ARI, septicaemia and UTI.

- Commonest complications encountered in the study are dehydration, followed sepsis, 
hypothermia and hypoglycemia.

- $27.4 \%$ of SAM children showed positive blood culture.

- The organisms isolated from blood cultures are Staphylococcus aureus followed by CONS, GDS, E.coli. and klebsiella

\section{Study Limitations}

1. This is a hospital based observational study and the true prevalence of SAM in the community could not be ascertained from it.

2. Many patients had already taken one or two of the commonly used antibiotics prior to recruitment. This may have lowered the detection rate of blood bacterial isolates and sensitive pattern.

\section{Sources of support or funding: None}

\section{Bibliography}

1. WHO. Guideline: Updates on the management of severe acute malnutrition in infants and children. Geneva: World Health Organization; 2013.

2. World Health Organization, Country Office For India; National Rural Health Mission (IN). Facility Based Care of Severe Acute Malnutrition: Participant Manual.(New Delhi): World Health Organization, Country Office for India; 2011 Mar. 119p

3. International Institute for Population Sciences (IIPS) and ICF. 2017. National Family Health Survey (NFHS-4), 2015-16: India. Mumbai: IIPS.

4. Bagga A, Tripathi P, Jatana V, Hari P, Kapil A, Srivastava RN, et al. Bacteriuria and urinary tract infection in malnourished children. Pediatr Nephrol 2003; 18:366370.

5. Bernal C, Velásquez C, Alcaraz G, Botero, J. Treatment of severe malnutrition in children: Experience in implementing the World Health Organization guidelines in
Turbo, Colombia. J Pediatr Gastroenterol Nutr 2008;46:322-328.

6. Kelsey D J Jones,JamesA Berkley,'Severe acute malnutrition and infection", Paediatr Int Child Health.2014;34(Suppl 1):1-29

7. Umma Abdullahi Idris, Wammanda D. Robinson, Jamilu A Faruk, Garba Dayyabu Gwarzo.'Prevalence of Bacteremia among Febrile Children with Severe Malnutrition in North Western Nigeria". Nigerian Journal of General Practice : Volume 16 i Issue 1 i JanuaryJune 2018

8. Mahgoub HM,Adam I.Morbidity and mortality in severe malnutrition among Sudanese children in New Halfa Hospital, Eastern Sudan.Trans R Soc Trop Med Hyg.2012;106:66-8.

9. Ashraf S, Javed MT, Abbas N, Aysha H, Hameed S (2001) Malnutrition in diseased children with reference to age, sex, socioeconomic status and area of living. Int J Agri Biol 3(4): 419-422

10. Berkley JA, Lowe BS, Mwangi I, et al. Bacteremia among children admitted to a rural hospital in Kenya. N Engl J Med 2005; 352(1).pp.39-47

11. Wiedermann U, Tarkowski A, Bremell T, Hanson L, Kahu H, Dahlgren U: Vitamin A deficiency predisposes to Staphylococcus aureus infection. Infect Immun. 1996, 64 (1): 209-214.

12. C. D. C. Christie, G. T. Heikens, and M. H. N. Golden, "Coagulase-negative staphylococcal bacteremia in severely malnourished Jamaican children," Pediatric Infectious Disease Journal, vol. 11, no. 12, pp. 1030-1036, 1992.

13. Piette, A. \& Verschraegen, G. (2009). Role of coagulase-negative staphylococci in human disease. Vet Microbiol 134, 45-54.

14. Uduak A. Okomo, Danlami Garba, AugustinE. Fombah, Ousman Secka." Bacterial Isolates and Antibiotic Sensitivity among Gambian Children with 


\section{JMSCR Vol||06||Issue||10||Page 1114-1120||October}

Severe Acute Malnutrition" International Journal of Pediatrics, Volume 2011, Article ID 825123

15. Alem A, Alemseged A, Getenet B, Genet $\mathrm{G}$ : "Bacteremia among severely malnourished children in Jimma university hospital, Ethiopia" Ethiop J Health Sci. Vol. 21, No. 3 November 2011

16. M.R.Prasanth, M.R. Savitha, H.N. Yashwanth Raju, M. Shanthi," Clinical Spectrum of severe acute malnutrition admitted to nutritional rehabilitation centre of a tertiary care hospital with special reference to incidence of bilateral pitting pedal oedema in children with severe acute malnutrition," International Journal Of Contemporary Pediatrics,2018 Sep; 5(5):1928-1932

17. Sharma, M. Lata "P1190 A study of malnutrition and associated infection in children in urban private Hospital in India”. Journal of Pediatric Gastroenterology \& Nutrition June 2004Volume 39-Issue p-S509.

\footnotetext{
Abbreviations

SAM Severe Acute Malnutrition

MUAC Mid Upper Arm Circumference

NFHS National Family Health Survey

NCHS National centre for health statistics

RTI Respiratory Tract Infection

UTI Urinary Tract Infection

GERD Gastroesophageal reflux disease

AGE Acute gastroenteritis

SES Socioeconomic status

CONS Coagulase negative staphylococcus

HIV Human Immunodeficiency Virus

AIDS Acquired immunodeficiency syndrome

TB Tuberculosis

CBC Complete blood count

CPS Comment peripheral smear

E.COLI Escherichia coli

ESR Erythrocyte sedimentation rate

GDS Group D streptococcus
}

SD Standard deviation

SCBMCH Sriram Chandra Bhanja Medical

College \& Hospital

SVPPGIP Sardar Vallabhbhai Patel Postgraduate Institute of Paediatrics 